\title{
Hippocrates as the Physician of Democritus *
}

\author{
By Owsei Temkin
}

The legends that began to form around Hippocrates in the Hellenistic period received novelistic treatment in a series of fictitious letters that constitute the major part of the so-called Pseudepigrapha of the Hippocratic collection $^{1}$. By the end of the Christian first century ${ }^{2}$ these letters probably had the form they still have today ${ }^{3}$.

They are grouped around two themes. The first (Letters 1-9) deals with the invitation to Hippocrates from the Persian king to stop the plague devastating the army, and with the physician's patriotic refusal to serve the enemy of Greece and his rejection of the riches and honors offered to him.

The second theme (Letters 10-23) ${ }^{4}$, loosely connected with the first, is the alleged insanity of Democritus, which his fellow citizens, the Abderites, wish Hippocrates to cure. In particular, Letters 10-17 present a coherent story beginning with the invitation and ending with the meeting of physician and prospective patient. I shall try here to narrate and briefly interpret this "story" as related in letters to and by Hippocrates ${ }^{5}$.

In the invitation by the senate and people of Abdera, Hippocrates is told that Democritus has become ill from too much wisdom and were he to lose his reason, the city might be abandoned ${ }^{6}$. The following are the symptoms as listed:

"He carries on, forgetful of all and foremost of himself, awake day and night, laughing at everything great and small, and counting all of life for nothing. One [person] marries, others trade, make public speeches, assume command, or serve as ambassadors, are voted into office, are sick, wounded, die but he laughs at it all, at those whom he sees downcast and sullen as well as those full of joy.

He also inquires into and writes about the things in the nether world, and he says that the air is full of images and that he listens attentively to the voices of birds. Moreover, often getting up at night, alone, he seems to sing songs softly, and he claims sometimes to go off into infinity and that there are innumerable Democrituses like himself. He lives on, his complexion having deteriorated together with his mind." 7

\footnotetext{
* Dedicated to my friend, Professor Jean Starobinski, on the occasion of his 65th birthday.
} 
These symptoms can be roughly divided into three categories. The undiscriminating laughter at everything, the strange ideas, and the insomnia and solitary behavior.

Though the Abderites realize that the acquisition of knowledge ${ }^{8}$ will mean more to Hippocrates, they also offer him money and glory in abundance. The city is in a turmoil and he will have all but dictatorial power if he comes. All of Greece, education itself, the future age - all need him, and he is urged not to fail Democritus and the city.

But the Abderites cannot forgo returning to their opinion that too much wisdom is the cause of Democritus' sickness, whereas the mass of the Abderites, "who have remained uneducated not only retain their common sense, but they who formerly were foolish, are sensible enough to judge the disease of a sage." ${ }^{9}$ Hippocrates must come and bring the proper remedies for curing madness.

Hippocrates accepts (Letter 11), praising the Abderites' solid support of Democritus and their realization that good men and wise counsel rather than towers and walls are the real bulwark of a city. But he is dismayed over being offered money. He will come in obedience to Nature, whose work man is and who wants him to restore the endangered piece of workmanship, and in obedience to the gods, by whose favor the arts have come into existence. He hastens to cure Democritus of his disease, if indeed it is a disease, and not an error of the Abderites as he would like it to be.

But medicine must remain a free art. Physicians who accept a fee enslave their profession and are likely to commit all kinds of knavery. The lust for money makes human life miserable. Would that all physicians united to cure this, a madness all the worse because it is even praised. "I believe that all diseases of the soul are severe madnesses that inflict certain opinions and phantasies upon reason, and that recovery consists in being purged by virtue."10

From the very first, Hippocrates has been doubtful that Democritus is sick, and in the next letter (12), to Philopoimen, who will be his host in Abdera, he inclines to the optimistic view that Democritus is not mad. Rather he manifests "an exceeding strength of soul in having no thought for children, wife, relatives, his property, or anything at all, and by day and night concentrating upon himself and living alone mostly in caves and isolated places, under the shadow of trees, on soft grass, or by gently flowing waters." "Usually," Hippocrates continues, "such things occur in melancholics; they sometimes too are silent, solitary, and fond of lonely places, shun people, taking a familiar face for a stranger." 11 
Hippocrates obviously elaborates the data given by the Abderites, establishing types: the strong, self-concentrated mind, and the behavior seen in melancholics. To this he now adds a third line: "Nor is it unreasonable that in those who are dedicated to study other thoughts are driven away by their exclusive disposition to wisdom." 12 As unruly servants become silent upon the sudden appearance of their mistress, so it is with the other desires of the soul, which are servants of things evil for man. Upon the sight of wisdom "the remaining affects are driven out like slaves." 13

There is still a fourth possibility, for the insane are by no means the only ones to yearn for caves and quiet. People who look down upon human affairs in their quest for calm (ataraxiē) ${ }^{14}$ let the body rest when their mind, overwhelmed by external troubles, wishes to regain strength. Then the mind surges upward within itself and looking around "beholds a country of truth" 15 in which there are no relatives or any of the things that cause trouble. These things do not dare to come near the inhabitants of this region: arts, virtues and skills, gods, demons, projects, maxims, a region where "the large vault of heaven is decked out with the restless stars." ${ }^{16}$ Perhaps wisdom has transported Democritus into this region. It is as if he had emigrated to a faraway place and no longer saw those in the city. His longing for solitude is taken for insanity. The Abderites in their preoccupation with money show that they do not understand him.

Possibly, the dwelling in the world of the stars is to account for Democritus' peculiar ideas; otherwise the four possibilities only envisage the melancholic recluse and three states which, though unusual, are not morbid. The whole diagnostic deliberation is related to the famous pseudo-Aristotelian problem (30.1) of the relationship of genius and melancholy ${ }^{17}$.

Hippocrates does not rule out the insanity of Democritus as medically impossible. But for reasons set forth in the next letter (13) he thinks of it as not only uncertain but very improbable.

This letter is addressed to his friend Dionysius whom he wants to come to Cos as his locum tenens, and who would also look after his wife. She will stay with her parents; nevertheless, being a woman, she needs suppervision which a friend whose judgment is not swayed by affection is better qualified to supply.

Hippocrates suspects that the whole city of Abdera may need treatment, a suspicion that contrasts with the Abderites' own view of the sanity of hoi polloi, the uneducated masses. He believes that Democritus, a highly educated sage, has been mistakenly dubbed insane from an excess of 
education. These laymen consider excessive, and therefore morbid, that which they themselves lack. To Hippocrates an excess of virtue is never harmful.

There is the matter of transportation, and Hippocrates next writes to Damagetes regarding the ship that will take him to Abdera (Letter 14). Time is pressing: he intends to cure the city which is ill because Democritus is ailing and accused (erroneously, he believes) of being insane. "He laughs constantly they say and does not stop laughing about any matter and this to them seems a sign of insanity." 18 At this point Hippocrates prudently advises his friends to follow a middle course, neither to laugh too much nor to appear gloomy too often. But though immoderate laughter is bad enough, persistent laughter at everything is much worse. He would like to point out to Democritus that grief and joy both exist in the universe and that in discarding one of them, i. e., grief, he fights the gods. It is not as if his laughter kept those close to him in good health. Hippocrates is not speaking of meaningless insane laughter; Democritus' indiscriminate laughter carries a sinister moral meaning ${ }^{19}$. "You laugh about the sick, you are delighted when they die, you cheer when you hear anything bad. How very wicked you are, Democritus, and far from wisdom. Or do you think that this is not evil? You are crazy ${ }^{20}$, Democritus, and in danger of yourself being an Abderite, whereas the city is the more reasonable." 21 The insanity of which Hippocrates speaks now is not on the same level as what he discussed before. It is a moral accusation thrown at the patient, not a detached diagnosis. But so far it is all imaginary. About all this, Hippocrates tells Damagetes, "I shall speak with greater accuracy when I am [in Abdera]." 22

Next (Letter 15) we hear of a dream that Hippocrates related to Philopoimen, in which Asclepius appeared to him when the ship was approaching Abdera. The god rejected his request to stand by him in the treatment of Democritus. That was not necessary, Hippocrates would be guided by a divinity both gods and humans recognized. It proved to be a beautiful woman who led him to the house where he was to stay. Tomorrow morning, so she promised, she would rejoin him at Democritus' place. Asked who she was, she said: "Truth, and the woman you see approaching is called Opinion, and she dwells with the Abderites." ${ }^{23}$ When awake, Hippocrates interpreted the dream to mean that Democritus did not need a physician and that "the truth of his being healthy abides with Democritus, whereas the opinion of his being sick dwells with the Abderites." 24 Hippocrates believes in the dream. In his opinion, medicine and divination are closely related, 
since both were fathered by Apollo. At any rate, Hippocrates feels that nothing untoward is going to happen.

In spite of his subjective certainty that Democritus is not mad and that purging drugs will not be needed, still it is necessary to be prepared for all eventualities as he writes to the herbalist Crateuas (Letter 16). And so he asks him for a goodly supply, giving him detailed directions about the quality of the plants, their location, and how to send them ${ }^{25}$. He urges speed; in medicine delay means endangering life. Hippocrates adds various reflections on therapy and its uncertainties, on the expectation of the patient who wants the impossible and on the difficult position of medicine between the patient in whom all is hidden and the art that is limited. All this necessitates being prepared for unforeseen eventualities. But he hopes that in Democritus wisdom will replace the most active and efficient drugs.

Hippocrates cannot forget the evils of money and the ten talents ${ }^{26}$ offered him by the Abderites, as if he were a mercenary rather than a physician. "If only, Crateuas, you could cut out the bitter root of the lust for money, so that nothing were left of it to shoot forth. Be confident that in purging their bodies we would [then] also purge people's ailing souls." 27

The meaning seems to be that once the lust for money is destroyed, all other vices disappear with it. There is then no more need for purging with virtue $^{28}$. Only such madness remains as is concomitant ${ }^{29}$ with somatic imbalance, for instance, a surplus of bile, which will respond to a somatic purge. The symptoms that Democritus presented pointed partly to melancholy, caused by black bile, partly to morally objectionable opinions. The question was whether he was insane in either of these senses.

The last letter (17), again addressed to Damagetes, to whom Hippocrates previously (Letter 14) had expressed doubts about Democritus' morality, begins with the answer. "It was as we conjectured, Damagetes. Democritus was not out of his mind, rather he scorned all things and recalled us to our senses and all human beings through us." 30

In Abdera, Hippocrates was welcomed by the whole city and upon his request was led to an elevated place from which Democritus' residence could be seen. "Democritus himself, in a coarse tunic, alone, barefoot, very pale and thin, his beard in need of trimming, was seated on a stone bench beneath a low spreading plane tree," 31 in an idyllic landscape, reminiscent of that described in Plato's Phaedrus. ${ }^{32}$

A book on his lap, others lying around him together with many dissected animals, Democritus was now writing, now meditating. Then he walked 
about, inspected the viscera of the animals and sat down again. Telling the Abderites, who were bewailing Democritus, to stay, Hippocrates approached him quietly and waited till he looked up and said: "welcome stranger." ${ }^{33}$ Introductions were exchanged in most civil terms, and Democritus offered Hippocrates the hospitality of his house. Though it was clear to him that Democritus was not deranged, he used this opportunity for a test ${ }^{34}$. He asked whether Democritus knew Philopoimen with whom he was staying: "Very well, you speak of the son of Dames, who lives at the fountain of Hermes." ${ }^{35}$ Democritus obviously was well oriented.

Hippocrates now wished to know what Democritus was writing. "On madness," was the reply. "Oh king Zeus, what an opportune answer you give to the city," ${ }^{36}$ Hippocrates exclaimed, almost giving the show away. "But what are you writing about madness?" 37

Democritus wished to know what madness was, how it originated in people, and how it could be relieved. He dissected animals to learn the nature and the seat of bile, explaining that its increase usually was the cause of insanity. No wonder that Hippocrates was enthusiastic about his approach that was in the best tradition of Hippocratic science. "By Zeus, Democritus, you speak truly and reasonably. So I consider you fortunate to have the benefit of so much quiet. To me this has not been given." 38

Democritus asks why quiet has been denied Hippocrates. This question marks a turning point in the interview. "Because," he explained, "land, children, money matters, diseases, deaths, slaves, weddings, or such things cut off my leisure." ${ }^{39}$ Whereupon Democritus laughs, and mocks, and then is silent. Hippocrates wants to know whether Democritus derides the good things or the bad ones that stand in the way of his leisure. Democritus laughs even more, to the great distress of the watching Abderites. Hippocrates insists on knowing the reason for the laughter. It was absurd to laugh at evils such as human death or diseases, as well as at definite goods such as weddings and festivals.

The situation is now that anticipated in the previous letter (14) to Damagetes, where Hippocrates had raised the question of moral insanity. Democritus promises that when Hippocrates has learned the explanation, he will accept the laughter as a therapy even for himself. Hippocrates, he says, distinguishes two causes of his laughter: good things and bad things. Yet he, Democritus, laughs at only one thing: at man, who squanders his life in a senseless way. He gives a long list exemplifying man's baseness, folly, and 
greed. People do not know themselves and so they bring upon themselves misfortunes about which he also laughs.

Hippocrates acknowledges that Democritus has given a truthful picture of human misery. But he sees human faults where Hippocrates sees misery arising from the necessities of life into which nature has put man, and from human ambition and optimistic outlook. Everybody starts things confident of a favorable outcome.

Democritus has lost patience and accuses Hippocrates of being obtuse. He is not laughing at those who know themselves and their temperament and are able to manage their affairs. To Hippocrates' optimism he opposes a misanthropic view. "From birth, man as a whole is a disease: as an infant useless, a supplicant for help; when growing up, reckless, unreasonable, in the hand of pedagogues; reaching his prime, presumptuous; past his prime, pitiable for he cultivated his own troubles by his thoughtlessness. Indeed, such is he who leapt out of the gory womb." 40

It is not necessary to follow the additional recital of human follies and misdeeds. Democritus' speech has long been recognized as a Cynic diatribe ${ }^{41}$. Its effect on Hippocrates is total surrender. "Most glorious Democritus, I shall carry back to Cos great gifts from all your hospitality, for you have filled me with much admiration for [your] wisdom. I return home as your herald, who has tracked down and discerned the truth about human nature, and I take from you the therapy for my thought." 42 He must leave now, but they will continue tomorrow and the following days. To the Abderites he says: "Gentlemen, my sincere gratitude for your summons. For I have met Democritus, the wisest man, and he alone is most capable to set people aright." 43

To Ancient readers, who were accustomed to think of the Abderites as fools, the outcome in favor of Democritus probably did not come as a surprise. Perhaps they were not surprised either to find Hippocrates, great physician though he was, no match for the philosopher. In Antiquity physicians were expected to be experts in somatic disease, and the story presented him as such as long as he was concerned with possible melancholy. Physicians were not expected to deal with moral philosophy, which became the decisive issue.

But the story with its abrupt conversion of Hippocrates (no further meeting was reported) and with his vow to become Democritus' herald, leaves a disquieting question. Democritus suggests that Hippocrates teach him medicine, that is, after Hippocrates has realized with what zeal and 
eagerness people waste their time, pursuing what is worth no more than a laugh ${ }^{44}$. What kind of medicine would he then teach and practice? Would he go on treating his patients and convert them? Would he convert mankind first and then treat what was worth treating? Or would he treat them and laugh? Retrospectively, Democritus condemned his own research on insanity as foolish. Insanity should be studied in man, not in animals ${ }^{45}$. Should Hippocrates also denounce Hippocratic medicine?

The question remains unanswered, which indicates that for the story Democritus is the dominant person. Nevertheless, it has a place within the Hippocratic legend in as far as some people believed that Democritus was his teacher. But we may ask, can a physician be a Cynic in the Ancient sense without becoming cynical?

\section{Notes}

${ }^{1}$ On the legend and the pseudepigrapha in general see Robert Philippson, «Verfasser und Abfassungszeit der sogenannten Hippokratesbriefe,» Rheinisches Museum für Philologie, N.F.77 (1928): 293-328; Ludwig Edelstein, article "Hippocrates," Pauly-Wissowa, Realencyclopädie der classischen Altertumswissenschaft, Supplementband 6 (1935): cols. 1290-1305; Wesley D.Smith, The Hippocratic Tradition, Ithaca and London: Cornell, 1979, pp.215-222; Dimitrios Th.Sakalis, «Beiträge zu den pseudo-hippokratischen Briefen,» in Formes de pensée dans la collection hippocratique: Actes du IV colloque international hippocratique (Lausanne 21-26 septembre 1981). Geneva: Droz, 1983, pp.499-514; Jackie Pigeaud, La maladie de l'âme, Paris: Les Belles Lettres, 1981, especially pp.441-477.

The latest edition of the letters is by Walter Putzger, Hippocratis quae ferunter epistulae ad codicum fidem recensitae, Wurzen, 1914 (Wissenschaftliche Beilage zum Jahresbericht des kgl. Gymnasiums in Wurzen, Ostern 1914). I am indebted to Professor Wesley D.Smith for having made this edition available to me. References will be to Putzger's edition (P.) and to that of Emile Littré, CEuvres complètes d'Hippocrate, vol. 9, Paris: Baillière, 1861, pp.308-429 (L.). Littré's edition also contains the other three pieces of the Pseudepigrapha: a decree of the Athenians, and two speeches by Hippocrates and his son, Thessalus, respectively.

2 A relatively late dating (end of the second century) by Fridolf Kudlien, article "Paetus," Pauly-Wissowa, Realencyclopädie des classischen Altertums, Supplementband 10 (1965), cols. 473-74, rests on the doubtful identification of the addressee and writer of Letters 1 and 2 with the associate of Alexander of Abonotichos. According to Sakalis, l.c. (above, n. 1), pp. 503 and 504, n.26, the name of the correspondent of Artaxerxes was Petos not Paitos.

3 Regarding Letter 19 see Hans Diller, «Die sogenannte 2. Fassung des 19. Hippokratesbriefes,» Quellen und Studien zur Geschichte der Naturwissenschaften und der Medizin 3.1 (1933), pp. 243-52; also Pigeaud l.c. (above, n. 1), pp. 454 and 469. 
${ }^{4}$ Of these, Lettres 18 and 20 belong to a different version; regarding letter 19 see above, n. 3 , Letters 21 and 23 are only loosely connected with the theme and Letter 22 not at all.

5 Other aspects, as well as a more detailed analysis of the first theme and a discussion of the legend as a whole will be treated in a different context.

${ }^{6}$ Letter 10; P. (ed. Putzger) p. 4, 23-24; L. (ed. Littré) p. 320, 15-17.

${ }^{7}$ Letter 10; P.pp.4,24-5,8. L.pp.320,17-322,7; cf. Pigeaud, l.c. (above, n. 1). pp.462-64.

${ }^{8}$ Ibid., P. 5, 10; L.p. 322, 11-12. I think that "acquisition of knowledge" here meets the sense of paideia, a broad term that is not easily translated.

9 Ibid.; P.p. 5, 26-28; L. p. 324, 13-16.

${ }^{10}$ Letter 11; P.pp. 6,22-7,2; L.p. 328, 6-8. The Greek text does not make it clear whether the cure by virtue refers to the "madnesses" (as I believe) or to the opinions and phantasies.

11 Letter 12; P.p. 7, 17-23; L.p. 330, 8-16.

12 Ibid.; P.p. 7, 23-24; L.p. 330, 16-18.

13 Ibid.; P.p. 8, 2; L.p. 330, 22-23. The remaining desires and affects are those besides the desire for study and wisdom.

14 Ibid.; P.p. 8, 3-4; L.p. 332, 2.

15 Ibid.; P.p. 8, 6; L.p.332, 5.

${ }_{16}$ Ibid.; P.p.8, 10; L.p.332, 10-12. Hellmut Flashar, Melancholie und Melancholiker in den medizinsichen Theorien der Antike, Berlin: de Gruyter, 1966, pp.69-70 speaks of «nahezu platonischer Weise.»

17 See Hellmut Flashar (trans.), Aristoteles: Problemata Physica, 3rd ed. Berlin: Akademie Verlag, 1983, pp. 711-722; Pigeaud, l.c. (above n.1) p. 453.

18 Letter 14; P.p.9, 19-20; L.pp. 336, 19-338, 1.

19 Cf. Pigeaud, l.c. (above n. 1), pp. 474-77.

20 Melangcholāis.

${ }^{21}$ Letter 14; P.p. 10, 4-7; L.p. 338, 14-18.

22 Ibid., P.p. 10, 7; L. p. 338, 18-19.

${ }^{23}$ Letter 15; P.p. 11, 2-4; L. pp. 340, 21-342, 3.

${ }^{24}$ Ibid; P.p. 11, 5-7; L. p. 342, 6-7.

${ }^{25}$ See Jean Starobinski, Histoire du traitement de la mélancolie des origines à 1900, Basel: Geigy, November 1960, pp. 16-18.

${ }^{26}$ Letter 16; P.p. 11, 20; L.p. 344, 4-5. See also Letter 11, P.p. 7, 11; L.p. 328, 4. Letter 10 had not mentioned any exact amount.

27 Ibid.; P.p. 11, 15-17; L. p. 344, 5-8.

${ }^{28} \mathrm{Cf}$. above $\mathrm{n} .10$ and the passage to which it refers.

${ }^{29}$ Cf. Pigeaud, l.c. (above n. 1) p. 475 for concomitant.

30 Letter 17; P.p. 13, 2-3; L.p. 348, 5-7.

31 Ibid.; P.p. 13, 17-19; L.p. 350, 9-12.

32 Plato, Phaedrus 230 B; Pigeaud, l.c. (above n. 1) p. 455.

33 Letter 17, P.p. 14, 15; L.p. 352, 20.

${ }^{34}$ Ibid.; P.p. 15, 2; L.p. 354, 14.

${ }^{35}$ Ibid.; P.p. 15, 3-4; L.p. 354, 16-17.

36 Ibid.; P.p. 15, 6-7; L.p. 356, 1-2.

37 Ibid.; P.p. 15, 8-9; L.p. 356, 4.

38 Ibid., P.p. 15, 14-15; L.p. 356, 12-14. 
${ }^{39}$ Ibid.; P.p. 15, 16-17; L.p. 356, 15-17.

${ }^{40}$ Ibid.; P.p. 20, 8-11; L.pp. 372, 18-374, 2.

${ }^{41}$ See Edelstein, l.c. (above n. 1) cols. 1303-1304. It is quite possible, as Edelstein thinks, that the story originated as a fusion of two themes: the recognition of Democritus as a genius rather than a madman, and the victory of Democritus over Hippocrates; cf. also Philippson, l.c. (above n. 1). But whatever its origin it was meant to make sense as a whole.

${ }^{42}$ Letter 17; P.p. 21, 16-19; L.pp. 378, 12-380, 2.

${ }^{43}$ Ibid.; P.pp. 21, 22-22,2; L.p. 380, 7-9.

${ }^{44}$ Ibid.; P.p. 16, 9-11; L.pp. 358, 22-360, 2.

${ }^{45}$ Ibid.; P.p. 20, 6-7; L.p.372, 15-17.

\section{Zusammenfassung}

Die Studie behandelt jenen Teil der sogenannten Hippokratesbriefe, der vom Besuch des Arztes bei Demokrit handelt. Der Philosoph fiel seinen Mitbürgern, den Abderiten, dadurch auf, daß er ohne Unterschied über alles lachte, merkwürdige Ideen äußerte, schlaflos war und sich von den Mitmenschen absonderte. Man hielt ihn für verrückt und rief Hippokrates zur Behandlung. Dieser schreibt vor seinem Besuch, daß solches Benehmen wohl bei Melancholikern vorkomme, aber auch die intensive Beschäftigung eines Denkers mit der höchsten Weisheit könnte sich so äußern. Nach seiner Unterredung mit Demokrit neigt er zur letzteren Ansicht. Anderseits erschien das unterschiedslose Lachen über Gutes und Böses anfänglich auch Hippokrates als moralischen Wahnsinn. Doch läßt sich der Arzt von dem Philosophen zu einer kynischen Anschauung bekehren, die im Grunde die Fortführung seiner ärztlichen Tätigkeit in Frage stellt.

Owsei Temkin

Institute of the History of Medicine

The Johns Hopkins University

1900 East Monument Street

Baltimore MD 21205

U.S.A. 


\section{Le personnage du médecin dans l'œuvre romanesque de Stendhal *}

A Jean Starobinski, médecin et stendhalien

Par Jean Théodoridès

Cette étude s'inscrit dans un ensemble qui n'a fait jusqu'ici l'objet d'aucun travail exhaustif, à savoir la place occupée par la médecine et les médecins dans la littérature française ${ }^{1}$.

Pour le $19^{\mathrm{e}}$ siècle ce thème est important en particulier en ce qui concerne Balzac $^{2}$ et Zola ${ }^{3}$.

Il n'est pas non plus négligeable chez Stendhal, comme nous allons essayer de le montrer.

Petit-fils d'un médecin grenoblois assez célèbre localement, le docteur Henri Gagnon (1728-1813), lui-même frotté de culture médicale et ayant connu en tant que consultant ou à titre amical de nombreux praticiens français et étrangers ${ }^{4}$, Stendhal n'a pas pour autant accordé une place importante à la profession médicale dans son univers romanesque.

Cependant de nombreux médecins traversent les romans stendhaliens et c'est à eux que sera consacré cet exposé.

Le premier: Armance (1827) a un fondement purement médical, son héros, Octave de Malivert représentant un cas pathologique qui a fait l'objet de nombreux commentaires.

Il s'agit en effet d'un impuissant dont l'infirmité était davantage liée à des troubles psychiques qu'à une malformation physique. Octave est un anxieux sujet à des crises de violence (il défenestre un domestique...) alternant avec des épisodes dépressifs qui le mèneront au suicide final.

Toutefois, par un paradoxe bien stendhalien, il n'y a pas dans ce roman de personnage médical y jouant un rôle important. Seul le docteur Duquerrel (médecin et chirurgien), nom forgé à partir de ceux de deux médecins réels: Dupuytren et Duquénelle ${ }^{5}$ apparaît çà et là. Il est à la fois le médecin de Madame de Malivert et de son fils Octave qu'il soigne après son duel avec le marquis de Crèveroche et guérit d'un «tétanos» quelque peu mythique. Mais aucun portrait, ni physique, ni moral n'est donné de ce praticien qui ne joue ici qu'un rôle d'«utilité».

* Communication présentée au Congrès International Stendhalien (Paris) le 26 avril 1983.

Gesnerus 42 (1985) 465-478 
Notons encore qu'une allusion faite aux chefs de deux sectes médicales rivales sous-entend peut-être les noms de Laennec et de Broussais qui ne sont cependant pas prononcés ${ }^{6}$. Dans Le Rouge et le Noir (1831) il est fait allusion à un vieux chirurgien-major de l'armée d'Italie retiré à Verrières qui était jacobin et bonapartiste. Il avait enseigné à Julien Sorel le latin, l'histoire et l'escrime et lui avait légué sa petite bibliothèque. Mais il demeure anonyme et ne joue aucun rôle dans l'intrigue du roman. Ce personnage a probablement été suggéré à Stendhal par un des médecins militaires de la campagne d'Italie qu'il avait connus personnellement et notamment Salmon ${ }^{7}$ ou Gonel.

Dans La Chartreuse de Parme (1839) n'apparaît pour ainsi dire aucun médecin. On sait cependant que le «pilotis» principal du personnage de Ferrante Palla est le célèbre docteur Giovanni Rasori (1766-1837) que Stendhal avait connu personnellement et qui était, comme Palla, médecin, poète et républicain ${ }^{8}$.

On ne trouve pas de personnage médical bien caractérisé dans les Chroniques Italiennes ou les Romans et Nouvelles où n'apparaissent que quelques «utilités».

Jusqu'ici nous n'avons considéré que des œuvres parues du vivant de Stendhal. Nous devons maintenant examiner les romans posthumes que sont Lucien Leuwen et Lamiel et c'est là que notre moisson sera la plus riche.

\section{Lucien Leuwen (1834-36)}

Plusieurs médecins sont mis en scène dans ce roman inachevé 9 . Considérons les suivant leur apparition chronologique dans l'ouvrage. Le premier cité est le chirurgien-major du régiment de Lucien stationné à Nancy. Il s'agit du chevalier Billars «sorte de charlatan assez bon homme, originaire des Hautes-Alpes». Sous ce nom à peine modifié, on reconnaît Dominique Villars (1745-1814) médecin et botaniste qui fut chirurgien à l'hôpital militaire de Grenoble et enseigna l'histoire naturelle á l'école centrale de cette ville où Stendhal fut élève ${ }^{10}$.

Et c'est par ce médecin militaire que Lucien va connaître un autre praticien de Nancy qui va jouer un très grand rôle dans la première partie du roman: le docteur Du Poirier.

Dans la conception «mythique» des personnages stendhaliens, très bien définie par G. Durand ${ }^{11}$ Du Poirier fait partie de la cohorte des adversaires 
du héros, des dragons et cerbères qui lui barrent la route. Du Poirier va en effet s'opposer à Lucien tant sur le plan politique (il est furieusement «légitimiste») que dans ses entreprises sentimentales.

Dans toute la partie du roman qui a pour cadre Nancy, Du Poirier va en effet tout faire pour contrecarrer l'idylle de Lucien avec Madame de Chasteller et ses manigances atteindront leur apogée avec le pseudo-accouchement de Bathilde. Du Poirier, bien que médecin ou peut-être précisément parce qu'il l'est apparaît comme un personnage antipathique, pervers et dénué à la fois de sens moral et de celui de l'éthique médicale.

Ses interventions professionnelles sont minimes et se bornent aux soins qu'il donne à Lucien lorsque celui-ci est blessé à la suite d'un duel ou souffre d'une attaque de «goutte volante» (chapitre VIII).

Par contre il se dépense beaucoup en intrigues de salons et fait du prosélytisme politique.

Comme tous les personnages stendhaliens, Du Poirier offre des traits physiques et de caractère appartenant à des personnages réels. Nous avons déjà signalé ailleurs ${ }^{12}$ plusieurs de ces «pilotis» sur lesquels il nous faut revenir.

Il est bien reconnu aujourd'hui que les évènements politiques et le contexte social évoqués dans Lucien Leuwen sont basés en grande partie sur des évènements réels survenus à Lyon (et accessoirement à Vienne) sous la Monarchie de juillet.

Un médecin que Stendhal a connu personnellement et à qui il faisait même à un moment l'honneur de figurer parmi ses amis est le docteur Gabriel Prunelle (1777-1853) d'origine dauphinoise et qui fut maire de Lyon de 1830 à 1834. Comme nous l'avons montré précédemment ${ }^{12}$ Prunelle a certainement servi de modèle d'une triple façon à Du Poirier à la fois sur le plan onomastique (la prune et la poire...) sur une ressemblance physique (aspect «beethovénien» de Prunelle) et d'après ses idées politiques (Prunelle fut loin d'être un libéral...).

Dans sa récente édition de Lucien Leuwen ${ }^{13}$ Madame A. M. Meininger a apporté un témoignage supplémentaire à l'appui de notre hypothèse en montrant que Prunelle était nommément cité dans un article de la Gazette (1834) utilisé par Stendhal. Le même auteur rappelle un autre «pilotis» de Du Poirier indiqué dans une note marginale de Stendhal précisant: «D’abord le physique de Salvi; mais celui de Rubichon vaut mieux.» L'éminente balzacienne et stendhalienne experte propose dans ce contexte «à partir du système de déformation de Stendhal sur les noms, un personnage réel». 
Il s'agit d'Antonio Maria Salvadori, médecin Italien de Roveredo (région de Trente) nommément cité dans le Journal de Beyle en date du 18 juin $1801^{14}$.

Ce curieux personnage, véritable «médecin conspirateur» comme l'est Du Poirier fut agitateur à Naples et en Calabre et espion du pape en Russie. Pendant près d'un an il exerça en tant que médecin militaire à Grenoble sous le nom francisé de Sauveur ${ }^{15}$.

Ajoutons qu'il réapparaît sous le nom de Frédéric Sauven dans un fragment de décembre 1837 intitulé Le Conspirateur ${ }^{16}$. Bien que ce dernier ne soit pas médecin c'est certainement Salvadori alias Sauveur qui en est le modèle, tant par son nom que par ses activités.

Mais il ne faut pas oublier un autre «pilotis» important mais non médical de Du Poirier, à savoir le publiciste grenoblois Maurice Rubichon (1766-1849) bien connu personnellement de Stendhal et dont les idées légitimistes furent utilisées pour dépeindre celles du médecin de Nancy.

Madame Meininger nous donne d'intéressants témoignages de contemporains sur ce curieux personnage qui avait déjà fait l'objet d'études de Dumolard ${ }^{17}$ et F. Rude ${ }^{18}$.

Rubichon n'était cependant pas médecin alors qu'il existe un député légitimiste qui l'était: il s'agit du docteur Ailhaud.

Joseph Antoine Gaspard Vincent Ailhaud (1784-1867), docteur en médecine (1813), résidait à Nyons (Drôme) dont il fut maire-adjoint (1821-1826), puis député légitimiste sous Louis-Philippe (1834-1837). Stendhal a-t-il pensé à ce dauphinois à la fois médecin, homme politique et légitimiste en campant le personnage de Du Poirier? Nul ne pourra hélas répondre à cette question. Feuilletant les tables de l'édition de Stendhal au Divan ${ }^{20}$, nous y avions lu le nom d'Ailhaud renvoyant à une lettre de Stendhal à Mareste (Trieste, 28 janvier 1831) signée de ce nom ${ }^{21}$.

Mais dans l'édition de la Pléiade de la Correspondance est donnée la lecture Arthaud, un des pseudonymes de Stendhal ${ }^{22}$. Le «pilotis» Ailhaud reste donc très conjectural.

Pour en revenir à Lucien Leuwen, très peu de détails y sont donnés sur la pratique médicale de Du Poirier. Balzac aurait décrit en détail son cabinet de consultation et sa clientèle comme il l'a fait pour le Dr. Bénassis dans Le Médecin de campagne 22 bis. Rien de tel chez Stendhal qui fait de Du Poirier (dont on ignore le prénom) un personnage abstrait dont seules les idées et le caractère l'intéressent. En ce sens il n'est que secondairement médecin et aurait pu avoir une toute autre profession. 
Pour le dépeindre, Stendhal n'a cependant pas hésité à recourir à des métaphores animales. Dès son apparition au chapitre VIII de la première partie du roman, il est défini comme étant «un être de la dernière vulgarité, et qui semblait fier de ses façons basses et familières», et Stendhal ajoute: «c'est ainsi que le cochon se vautre dans la fange avec une sorte de volupté insolente pour le spectateur». Plus loin le docteur a «la physionomie d'un renard alerte», des «petits yeux gris comme ceux d'une hyène» et une «physionomie de sanglier».

Nous ne souscrivons pas pour autant à la remarque faite par le professeur H. Imbert (Colloque sur Lucien Leuwen, Paris février 1983) qui voit dans ces métaphores une composante balzacienne. En effet même si Balzac utilise davantage ce procédé ${ }^{23}$ que Stendhal, ce dernier s'en est souvent servi aussi ${ }^{24}$.

Il y a un autre épisode politico-médical dans la seconde partie (parisienne) de Lucien Leuwen où Du Poirier est absent. Il s'agit de l'affaire Kortis que l'on peut ainsi résumer: Lucien est chargé par le comte de Vaize, ministre de l'Intérieur d'obtenir le silence d'un agent provocateur, un policier (déguisé en ouvrier) nommé Kortis ${ }^{25}$ grièvement blessé d'un coup de fusil dans le ventre en essayant de désarmer une sentinelle gardant un pont.

L'évènement réel, le "petit fait vrai» qui inspira à Stendhal cet épisode eut lieu à Lyon le 8 juin 1834 et le policier s'appelait Corteys.

Lucien se rend donc à l'hôpital où le blessé est soigné dans le service d'un chirurgien «une espèce de colosse nommé Monod qui ne lit que le Courrier français au café près de l'hôpital».

Comme nous l'avons indiqué précédement, un chirurgien portant ce nom a réellement existé ${ }^{26}$. Il s'agit du docteur Frédéric Clément Constant Gustave Monod (1803-1890) qui habitait 20 rue Bleue et était donc le voisin de Madame Azur (Alberthe de Rubempré), cousine du peintre Delacroix et amie plus qu'intime de Stendhal qui en entendit peut-être parler par elle.

Pour revenir au roman, de Vaize avait exposé à Lucien ses craintes qu'on n'empoisonnât le blessé pour l'empêcher de parler et désirait que l'on obtienne son silence, dût-il être monnayé. Des tentatives plus radicales dans le même but sous la forme d'un empoisonnement par l'opium avaient été entreprises par un certain général N... dont dépendait Kortis.

Mais le Dr. Monod qui avait un sens de l'éthique médicale plus développé que Du Poirier avait reçu par un coup de poing l'émissaire dudit général.

Une fois à l'hôpital Lucien assiste à une consultation donnée par sept médecins et chirurgiens au chevet du blessé ${ }^{27}$. Cinq d'entre eux conclurent «à 
la mort possible à chaque instant, et certaine avant deux ou trois jours» vu la gravité de la blessure.

L'un d'eux (Lucien pensa qu'il s'agissait du «coquin gagné par le général N...») proposa l'opium et un autre «une saignée abondante au pied, pour prévenir l'hémorragie dans les entrailles», ce que le Dr. Monod suivi par les autres médecins repoussa de toutes ses forces, une telle saignée n'ayant selon lui «qu'un effet hors de doute, celui d'ôter la parole au blessé».

Sa mort survenue fort à propos une semaine après, sans qu'il ait parlé, mit un point final à cette sordide affaire.

\section{Lamiel (1839-42)}

Dans ce roman inachevé apparaissent plusieurs médecins dont le plus important est le docteur Sansfin sur lequel nous reviendrons plus loin.

Nous évoquerons d'abord rapidement les autres praticiens qui apparaissent à l'occasion de deux épisodes médicaux: la faiblesse oculaire de la duchesse de Miossens, la maladie de Lamiel.

Dès la première rédaction du roman ${ }^{28}$ (octobre 1839) on lit au chapitre I que les yeux de la duchesse «s'entourèrent de quelques rides «et qu'elle fut au désespoir». Elle expédia aussitôt un courrier à Paris et fit venir «le plus célèbre oculiste». Celui-ci vint à Carville et après avoir examiné la patiente pensa qu'il s'agissait d'amorphose et «conseilla à la duchesse de prendre une lectrice» pour ne pas se fatiguer les yeux.

Dans la seconde rédaction du roman (janvier-février 1840) cet épisode a lieu au chapitre IV et bénéficie d'un beaucoup plus long développement. Le nom de l'oculiste est précisé: il s'agit de M. de la Rouze (Stendhal avait écrit avant un autre nom biffé commençant par Del.) ${ }^{28 b i s}$

Nous ne reviendrons pas ici, en ayant déjà traité ailleurs ${ }^{29}$, sur les points suivants:

1. Le nom du praticien qui doit peut-être être lu «La Ronze» (par allusion au nom d'une localité dauphinoise: La Ronzie) qui est par ailleurs l'anagramme presque parfaite de (F)orlenza (ou Forlenze) oculiste italien fixé à Paris et cité par Stendhal dans Racine et Shakespeare.

2. Le terme d'amorphose est voisin phonétiquement de celui d'amaurose qui désignait à l'époque le syndrome connu aujourd'hui sous le nom de «décollement de la rétine». ${ }^{29 b i s}$ 
3. Les médicaments recommandés par l'oculiste à la duchesse (pilules à base de mie de pain et de coloquinte) semblent relever de la médecine psychosomatique.

La maladie de Lamiel est évoquée au chapitre $\mathrm{V}$ de la seconde rédaction (1840). Sa nature n'est pas précisée, mais justifie l'appel de plusieurs médecins dont le premier est le docteur Buirette, de Mortain. On appelle ensuite un médecin de Paris nommé Duchâteau, «sorte de Lovelace de faubourg» qui n'examina la malade que six minutes, "prescrivit un traitement absolument insignifiant» et repartit au bout de trois jours. Arriva enfin un troisième praticien de Rouen, nommé Derville (nom déjà utilisé dans Le Rouge et le Noir) qui déclara que Lamiel «n'avait pas six mois à vivre».

C'est alors que la duchesse se décida à faire appel à Sansfin dont il faut maintenant parler en détail.

Ce personnage est en effet présent dans la totalité des ébauches de Lamiel et prend de plus en plus d'importance dans les dernières à tel point que le roman eût presque pu sans inconvénient porter son nom au lieu de celui de l'héroïne.

V. del Litto évoque ${ }^{30}$ «les fragments de quatre ou cinq romans ayant pour personnage central d'abord Lamiel, héroïne de moins en moins vraisemblable, et de qui l'auteur se détache graduellement, ensuite le docteur Sansfin».

Dans ses diverses esquisses Stendhal donne de nombreuses précisions sur ce personnage: physiquement, il est «horriblement bossu», a de «beaux yeux, malheureux à la juive» (notons ce dernier qualificatif car il a, nous le verrons plus loin, une certaine importance).

Sa tête est «ornée d'une magnifique barbe noire» (ailleurs elle est blonde). Il est né tantôt en 1790, 1800, 1804, 1805, 1812, 1815, est le fils unique d'un riche fermier, ailleurs d'un conventionnel, qui lui laissa mille écus de rente et il s'était fait médecin pour apprendre à se soigner.

$\mathrm{Au}$ point de vue professionnel il a un certain talent digne de celui du docteur J.L.Prévost de Genève ${ }^{31}$, un des médecins les plus appréciés de Stendhal et est d'ailleurs qualifié d'«excellent» médecin. Dans une autre esquisse il devient député.

Stendhal précise par ailleurs qu'il gagne entre 7000 et 8000 francs par an ${ }^{32}$. Ailleurs il est dit qu'il avait 6000 livres de rente et triplait ce revenu par son état.

Quant à son caractère, Sansfin est partagé entre sa haine qui fait souffrir sa vanité et celle-ci qui fait souffrir sa haine. 
Il a de plus un côté donjuanesque, se vantant publiquement de ses conquêtes féminines et cherchant à séduire aussi bien la duchesse, femme mûre, que le tendron qu'est Lamiel.

Le côté peu scrupuleux de Sansfin va apparaître au chapitre V (rédaction de 1840) lorsque la duchesse de Miossens se décide à le faire appeler pour soigner Lamiel.

Au lieu de lui prescrire des remèdes devant la guérir, le machiavélique Sansfin lui en fait prendre d'autres «qui devaient redoubler tous les accidents de la maladie» et notamment certaines pilules «hérö̈ques». Mais voyant que malgré tout elle ne va pas plus mal et voulant la maintenir sous son influence, il obtient d'elle qu'elle mette dans sa bouche une petite éponge imbibée du sang d'un oiseau fraîchement tué afin de simuler des crachements de sang.

Ce simulacre d'une affection pulmonaire (phtisie) peut être mis en parallèle avec le pseudo-accouchement de Mme de Chasteller dans Lucien Leuwen et montre que pas plus que Du Poirier, Sansfin n'avait le sens de l'éthique et de la probité médicales qu'il trahissait par une telle manière d'agir.

Dans le même chapitre on voit le rusé docteur mettre sa clientèle en garde contre les premiers froids qui «peuvent répercuter sur la poitrine les humeurs que la transpiration insensible faisait disparaître et beaucoup de phtisies n'ont pas eu d'autre cause».

On peut se demander si Stendhal n'a pas forgé à plaisir cette explication fantaisiste de l'étiologie des affections tuberculeuses plus digne des médecins de Molière que des ceux de son époque.

Ailleurs (chapitre III de la rédaction de 1840) on voit Sansfin appelé auprès d'un «beau jeune homme de cinq pieds six pouces et qui venait, à peine âgé de vingt-cinq ans, d'avoir une belle et bonne attaque d'apoplexie».

Et Stendhal d'ajouter:

«Le docteur passa la nuit auprès de lui, et, tout en lui appliquant le traitement convenable, il eut le plaisir de voir cet être si beau mourir vers la pointe du jour.»

L'oraison funèbre qu'il prononça ensuite se réduisit à cette seule phrase: «Voilà un beau corps vacant...; pourquoi mon âme ne peut-elle pas y entrer?»

Et Stendhal d'épiloguer:

«Le docteur, fils unique d'un fermier enrichi par les biens nationaux, s'était fait médecin pour savoir se soigner. la récompense d'une activité souvent pénible pour sa faible santé était de voir mourir de beaux hommes et d'effrayer le petit nombre de jolies malades que le pays fournissait de façon à ce qu'elles désirassent sa présence avec passion». 
Revenons sur d'autres aspects du caractère de Sansfin. C'est aussi un homme violent qui peut se laisser aller à des réactions incontrôlées. Il est souvent armé de son fusil et a la détente facile n'hésitant pas à tirer avec du petit plomb sur de mauvais plaisants ou à tuer son chien désobéissant. Dans un autre épisode tardif (1842) intitulé «coup de poignard donné par un bossu», il poignarde, heureusement sans le tuer, un de ses rivaux auprès des beaux yeux de Lamiel.

Stendhal a-t-il pensé à un personnage réel en créant cette curieuse figure?

Très peu de «modèles» le concernant ont été jusqu'ici proposés. Seul a été rappelé le bossu Tourte connu par Stendhal dans son enfance pour ce qui est de son infirmité.

Le curieux nom de Sansfin n'a suscité, à notre connaissance qu'une seule tentative d'explication: celle de J.P. Weber ${ }^{33}$ qui y voit une allusion au supplice moral auquel le condamne sa perversité et qui est «sans fin»...

Mais aucun médecin réel n'a été jusqu'ici proposé comme modèle de Sansfin.

Or il y a une indication donnée par Stendhal lui-même qui nous semble avoir une certaine importance: c'est celle suivant laquelle Sansfin avait de «beaux yeux, malheureux à la juive».

Et nous pensons immédiatement à un médecin juif bien connu de Stendhal dont il était une «relation intime» (H. Martineau): le docteur David Ferdinand Koreff (1783-1851).

Beyle l'avait connu en 1828 chez G. Cuvier, lui reconnaissait beaucoup d'esprit et l'avait même consulté pour sa santé en 1833. Dans les Privilèges de 1840 il souhaitait au «privilégié» «l'esprit de M. Koreff (le médecin juif)». ${ }^{34}$ Or ce texte est contemporain de la rédaction de Lamiel.

Un autre passage de ce dernier roman appuyerait aussi ce «pilotis»: c'est celui où il est dit que Sansfin soumettait la duchesse de Miossens à l'«horrible magnétisme de son éloquence infernale». Or il se trouve que Koreff était précisément un adepte convaincu du magnétisme animal qui consistait à mettre les malades dans un état de somnambulisme et de léthargie ${ }^{35}$. C'est en somme un procédé thérapeutique précurseur de la psychanalyse. D'autre part, sans être à proprement parler bossu, Koreff était physiquement assez difforme. la comtesse Dash écrit dans ses Mémoires ${ }^{36}$ qu' $^{\prime}$ il avait du polichinelle dans les traits» et «une apparence presque grotesque».

Mais il ne faut pas oublier que, comme tout personnage stendhalien, Sansfin a des modèles multiples à commencer par Stendhal lui-même.

En effet, «l'enseignement fourni par Sansfin (à Lamiel) dérive en ligne 
droite de celui donné à Pauline Beyle par son frère» (R.Bolster) ${ }^{37}$ et il est hors de doute que Stendhal a donné au docteur beaucoup de ses propres traits de caractère.

Arrivé à ce point de notre exposé il nous reste à poser la question de savoir pourquoi dans deux de ses romans écrits à la fin de sa vie, Stendhal a donné une place importante à des médecins présentés sous un jour peu flatteur.

Une réponse partielle à cette interrogation se trouve dans un de ses projets beaucoup plus ancien puisqu'il date de 1804, à savoir celui d'écrire une comédie en un acte et en prose intitulée Les Médecins et inspirée de $L a$ Finta Ammalata de Goldoni.

Mais comme la plupart des projets «scéniques» de Beyle, celui-ci constitue une vélléité dont la réalisation concrète ne se fit jamais. Il en a néanmoins rédigé un plan qui contient des éléments significatifs ${ }^{38}$.

Stendhal reprochait surtout à la médecine de son époque d'être tributaire de divers «systèmes» où la théorie l'emportait sur l'observation directe. Ainsi celui de Brown qu'il cite en compagnie d'Erasme Darwin (le grand-père de Charles Darwin):

«Montrer une consultation. Ils disputent beaucoup sur les systèmes des médecins et parlent très peu de la malade. Savoir les noms et les principes des systèmes. Brown, Darwin».

Notons au passage que le même thème sera traité magistralement une vingtaine d'années plus tard par Balzac dans La Peau de chagrin où est décrite une consultation par les sommités parisiennes de l'époque (Dupuytren, Récamier, Magendie) au chevet de Raphaël de Valentin.

Pour en revenir au projet de pièce sur Les Médecins, il convient de rappeler qu'il trouva chez Stendhal son origine dans ses rencontres avec deux médecins, son presque homonyme Gaspard-Laurent Bayle (1774-1816) qu'il avait consulté pour sa santé et également Urbain-Philippe Salmon déjà cité précédemment.

Mais il y a, semble-t-il, une autre raison complémentaire des motivations précédentes pour laquelle Stendhal a dépeint dans Lucien Leuwen et Lamiel, œuvres de la fin de sa vie, des médecins sous un jour peu sympathique.

C'est qu'à partir de 1831 sa santé commença à décliner. Déjà affligé de goutte, la gravelle (lithiase urinaire) le tourmenta et l'obligea à consulter le docteur Jean-Louis Prévost, à Genève.

En outre il est couramment admis aujourd'hui que Stendhal souffrait d'hypertension comme l'attestent divers symptômes qui s'accentuèrent à 
partir de 1830: migraines, palpitations, congestion du visage et qui justifièrent une nouvelle consultation chez Prévost en juin $1838^{39}$.

Jusqu'alors Stendhal avait eu recours à un très grand nombre de praticiens qui l'avaient fort médiocrement soigné. Il en verra d'ailleurs encore d'autres, en Italie (Séverin, Dematteis, Dalbret, Alertz) tout aussi incompétents.

Les conseils et prescriptions de Prévost étaient justifiés et judicieux mais arrivaient trop tard.

Et l'on comprend que l'écrivain fort déçu du peu de compétence des divers médecins qu'il avait consultés ait pris un malin plaisir à en faire figurer deux: Du Poirier et Sansfin dépeints sous des traits quelque peu caricaturaux dans ses dernières œuvres.

Et, dans la même optique, on comprend très bien que Koreff qu'il avait consulté sans aucune amélioration de son état de santé ait un peu servi pour camper Sansfin.

L'état de santé du consul de France à Civitavecchia devait graduellement empirer: les migraines redoublèrent avec des troubles de la parole et l'on s'achemina irrémédiablement vers une issue fatale: le $1^{\mathrm{er}}$ janvier 1840 il eut une première attaque («tombé dans le feu en corrigeant la $35^{\mathrm{e}}$ page de Lamiel»), le 15 mars 1841 il en eut une seconde beaucoup plus sérieuse avec perte de connaissance. Le 5 avril suivant il écrivait à Di Fiore: ${ }^{40}$

«Je me suis colleté avec le néant», et ajoutait cette phrase qui nous semble capitale: «Croyant peu à la médecine et surtout aux médecins, hommes médiocres, je n'ai consulté qu'au bout de six mois d'atroces migraines». Et il manifestait à son ami l'intention d'écrire à «l'excellent M. Prévost de Genève». Le désir d'aller le consulter à nouveau est exprimé dans deux autres lettres: l'une à Guizot du 9 août $1841^{41}$ et l'autre à R. Colomb du 8 octobre ${ }^{42}$.

Stendhal revit Prévost pour la dernière fois, à Genève, le 4 novembre de la même année. Mais l'éminent praticien ne pouvait plus rien pour la santé de son malade devenu son ami. Le 22 mars 1842 une troisième attaque devait terrasser Stendhal qui s'éteignit le lendemain dans une chambre d'hôtel, dernière demeure idéale pour l'éternel voyageur qu'il avait été et dont nous honorons aujourd'hui la mémoire. 
${ }^{1}$ Le seul ouvrage sur la question est celui de R. Cruchet, La Médecine et les médecins dans la littérature française, Louisiana, 1939, $296 \mathrm{p}$. très sommaire pour ce qui est du $19^{\mathrm{e}}$ siècle. Il en est de même de celui de L.Binet et P. Vallery Radot, Médecine et Littérature, Paris, Expansion sci. fr. 1965, $267 \mathrm{p}$.

${ }^{2}$ Cf. M. Le Yaouane, Nosographie de l'Humanité Balzacienne, Paris, Maloine 1959.

${ }^{3}$ Cf. H. Martineau, Le roman scientifique d'Emile Zola, la Médecine et les Rougeon-Nacquart, Paris, Baillière, 1907, 272 p.

${ }^{4}$ Cf. J. Théodoridès, Stendhal du côté de la Science, Aran, Grand Chêne, 1972 (p. 101-230).

${ }^{5}$ Cf. A. Jacquinet et J.Théodoridès, Deux allusions médicales rémoises dans l'œuvre de Stendhal, Actes $95^{e}$ Congrès Soc. sav. Reims 1970, Sect. Sci., II, Paris, 1975, 121-130.

${ }^{6}$ Cf. C. Dédeyan, Laennec et les écrivains de son temps in: Laennec, 1781-1826, Rev. Pal. Découv. Paris, $N^{o}$ spécial 22, 1981, 245-260.

${ }^{7}$ Cf. F.F.Mars et J.Théodoridès, Un savant angevin de l'époque révolutionnaire: UrbainPhilippe Salmon (1768-1805), médecin militaire, géologue et ami de Stendhal, C.R.93e Congrès Soc. sav. Tours 1968, Sect. Sci., II, Paris 1971, 103-125.

${ }^{8}$ Cf. B. Pincherle, Lo stendhalesco dottor Rasori in: In compagna di Stendhal, Milano 1967, 81-120.

${ }^{9}$ Comme le fait remarquer A.M. Meininger dans son introduction à l'édition de Lucien Leuwen, Paris, Impr. nat. 1982, 2 vols. (I, 9-10) on peut en fait considérer ce roman comme presque achevé.

${ }^{10}$ Cf. J.Théodoridès, L'œuvre médicale de Dominique Villars (1745-1814), C.R. $108^{e}$ Congr. Soc. sav. Grenoble 1983, Sect. Sci., IV, 99-113.

${ }^{11}$ G. Durand, Le décor mythique de la Chartreuse de Parme, Paris, Corti, 1961, 251 p.

12 J.Théodoridès, Une source de «Lucien Leuwen»: le docteur Prunelle modèle probable du docteur Du Poirier, Stendhal Club, No 88, 1980, 315-328.

${ }^{13}$ op. cit. note $9, \mathrm{I}, 35$.

${ }^{14}$ Edit. Cercle bibl. XXVIII, 15-16.

${ }^{15}$ F. Michel, Fichier stendhalien, 3, 360-361.

${ }^{16}$ Edit. Cercle bibl. XLIX, 70.

${ }^{17}$ H. Dumolard, Un personnage de «Lucien Leuwen», le véritable docteur Du Poirier in: Autour de Stendhal, Grenoble, Arthaud 1932, 95-121.

${ }^{18}$ F. Rude, Un «prophète du passé» trop oublié: Maurice Rubichon in: Souvenirs de famille, Yemeniz, Rubichon, Bruysset, Lyon 1967, 18-33.

${ }_{19}$ Nous devons ce renseignement à l'obligeance du Prof. J.Léonard (Rennes), auteur de: La Médecine entre les pouvoirs et les savoirs, Paris, Aubier, 1981 (cf. p. 209).

${ }^{20}$ Stendhal, Table alphabétique des noms cités dans ses auvres, Paris, Le Divan 1937, I, p. 231.

${ }^{21}$ Stendhal, Correspondance, Paris, Le Divan 1934, VII, 98.

${ }_{22}^{2}$ Tome II, 241.

22bis Stendhal tout en écrivant Lucien Leuwen lisait ce roman de Balzac (cf. Euvres intimes, édit. Pléiade, II, 239-241; Journal litt., Cercle bibl. XXXV, 189, 213).

${ }^{23}$ L. F. Hoffmann, Les métaphores animales dans le Père Goriot, Année Balz. 1963, 91-105.

${ }^{24}$ G. Merler, La description physique des personnages dans les œuvres romanesques de Stendhal, Thèse Doct. Lettres Québec, 1967, No 1089, 590 p. dact.

${ }^{25}$ K. Furuya, Stendhal et l'affaire Corteys, Rev. Univ. Tokyo, 1967, 203-216.

${ }^{26}$ Stendhal du côté de la Science, op. cit. 214-215. 
27 Madame Meininger (Lucien Leuwen op. cit. note 9, II, 341) voit dans le «jeune médecin portant un nom bien connu dans les sciences» qui «écrivait le procès-verbal sous la dictée de Leuwen» une allusion à un neveu de G. Cuvier.

28 Nous avons utilisé l'édition de Lamiel par V.del Litto, Cercle bibl. XLIV qui en donne les rédactions successives.

28bis Madame A.M. Meininger dans sa toute récente édition de Lamiel, Folio $\mathrm{N}^{\circ} 1462$, Paris 1983, p.331 suppose que ce nom barré pouvait désigner les oculistes parisiens réels Delabarre ou Delachanterie, mais il peut aussi s'appliquer à Delaronze écrit en un seul mot. Précisions en outre que les Delabarre (père et fils) étaient dentistes et non oculistes. Si le «Del.» ne désigne pas Delaronze, c'est De La Chanterie (qui s'écrit en trois mots) qui paraît le «pilotis» le plus probable. Il exerçait 7 rue de la Monnaie et était connu par les affiches représentant un ceil traversé d'un pieu dont il faisait couvrir les murs de Paris (Cf. C. Sachaile, Les médecins de Paris jugés par leurs æuvres, Paris 1845, p.225).

${ }^{29}$ Stendhal du côté de la Science, 226-227; Stendhal et l'ophtalmologie, Stendhal Club, No 98 , 1983, p. 320-327.

29bis Pour A.M. Meininger (cf.op.cit.note $28^{\text {bis }}$ supra) Stendhal se serait rappelé le terme d'amorphie: désordre dans la conformation» (Littré) en raison de ses propres problèmes oculaires.

30 V.del Litto, Lamiel, pages inédites, St. Cl., No 1, 1958, 3-8.

${ }^{31}$ J. Théodoridès, Jean-Louis Prévost médecin et ami de Stendhal, Ibid. No 42, 1969, 177-189.

$32 \mathrm{~J}$. Léonard a rappelé cette indication dans La vie quotidienne du médecin de province au $X I X^{e}$ siècle, Paris, Hachette 1977, 105.

${ }^{33}$ J.P. Weber, Stendhal, les structures thématiques de l'œuvre et du destin, Paris, SEDES 1969, 491.

${ }^{34}$ V.del Litto, Un texte capital pour la connaissance de Stendhal: «Les Privilèges», Stendhal Club; No 13, 1961, 3-20.

35 A. Doyon et Y.du Parc ont montré dans leur ouvrage: De Mélanie à Lamiel. Aran, Grand Chêne 1972, à quel point Stendhal s'était servi des traits physiques et moraux de son ancienne maîtresse Mélanie Guilbert (Louason) pour camper le personnage de Lamiel. Or il se trouve qu'à la fin de sa vie, Mélanie devenue Madame de Barkoff était tombée sous la coupe d'un médecin charlatanesque, le docteur Nicolas Frapart (1792-1842) adepte du magnétisme animal, de la phrénologie et de l'homéopathie. Il est cependant très improbable que Stendhal ait eu vent de ces faits que nous tenions néanmoins à rappeler car ils constituent un nouvel exemple selon lequel la réalité peut dépasser souvent la fiction.

${ }^{36}$ Comtesse Dash, Mémoires des autres, V, 27-28 (cités par H. Martineau, Petit dictionnaire stendhalien, Paris, Le Divan 1948, 275.

37 R. Bolster, Les héroïnes rebelles de Stendhal, Stendhal Club, No 41, 1968, 21.

38 Théâtre, édit. Cercle bibl. XLIII, 11.

${ }^{39}$ Les meilleures études sur la pathologie de Stendhal sont les suivantes: X. Rousseau, Notes médicales sur Stendhal, Paris, Jouve 1938, 80 p. - E.Abravanel; Notes sur la santé de Stendhal Revue Thér. (Berne) 15e année, 1958, No 4, t.à.p. 15 p. - R.Soupault, Stendhal intime, Paris, Sept couleurs 1975, 324 p. Il faut mentionner aussi quoique plus général l'article de P. Chainet, Stendhal ou le mal milanais, Hist. de la Méd. $12^{\mathrm{e}}$ année, No 4, 1963, 12-31 où l'on trouve une amusante définition de Sansfin: «Le docteur Sansfin est le parfait médecin, le docteur Knock, le moderne disciple d'Hippocrate. Stendhal ne nous présente 
dans aucune de ses œuvres, avec une telle virtuosité, un docteur en médecine. Le docteur Sansfin est un étrange précurseur, c'est le Machiavel de la médecine moderne.»

${ }^{40}$ Correspondance édit. Pléiade, III, 434.

${ }^{41}$ Ibid. 489.

${ }^{42}$ Ibid. 4.98.

\section{Summary}

The character of the physician in Stendhal's novels

Grandson of a physician (Dr. H. Gagnon), himself well informed about medical questions and having personally known several prominent French and foreign physicians of his time, Stendhal has however not given in his novels an important place to the medical profession,

In Armance, Le Rouge et le Noir, La Chartreuse de Parme, there are few medical characters.

On the contrary in Lucien Leuwen and Lamiel published posthumously, two physicians: Dr. Du Poirier and Dr. Sansfin play a leading part as advisers of the heroines (Madame de Chasteller, Lamiel) to whom they dictate their behavior.

To describe these two ambitious, careerist and reactionary characters, Stendhal has taken as models some French physicians mingled with politics during the Restauration and the July Monarchy whom he might have known personally and for whom several names are suggested here.

J. Théodoridès

16 Sq. Port Royal

F-75013 Paris (France) 